\title{
Wirksamkeitsvermutungen in Bezug auf das Unterrichtshandeln bei Absolventen einer allgemeinpädagogischen Lehrerfortbildung
}

\author{
Tobias Jaschke
}

Eingegangen: 2. Februar 2016 / Angenommen: 10. Mai 2016 / Online publiziert: 9. Juni 2016 (C) The Author(s) 2016. This article is available at SpringerLink with Open Access

Zusammenfassung Die Wirksamkeit von Lehrerfortbildungen ist seit einigen Jahren verstärkt Gegenstand der empirischen Bildungsforschung. Im vorliegenden Beitrag wird eine explorative Interviewstudie $(N=3)$ vorgestellt, in der Absolventen einer allgemeinpädagogischen Lehrerfortbildung Selbstauskünfte zu ihren Wirksamkeitsvermutungen geben. Untersucht wird die Frage, welche subjektiv wahrgenommenen Auswirkungen die absolvierte Lehrerfortbildung auf das Unterrichtshandeln der beteiligten Lehrpersonen hatte. Unterrichtshandeln wird dabei als Teilbereich des Lehrerhandelns konzeptualisiert und operationalisiert sich in dieser Studie in der Art und Weise der Planung und Durchführung des mathematischen Regelunterrichts der Befragten. Die Ausführungen der Interviewpartner weisen primär auf punktuelle Veränderungen im methodischen Bereich hin, während substanzielle didaktisch-inhaltliche Auswirkungen der Lehrerfortbildung auf die Unterrichtsplanung und -durchführung kaum zu beobachten sind. Zwar kommt es bei den Teilnehmerinnen und Teilnehmern offenbar zu einer ,,aufgefrischten“ Sensibilisierung ihrer pädagogischen Haltung; der eigenständige Transfer der Fortbildungsinhalte in den Fachunterricht ist aber erkennbar problematisch. Insgesamt weisen die Ergebnisse darauf hin, dass es erstens sinnvoll sein kann, allgemeinpädagogische Lehrerfortbildungen fachspezifisch $\mathrm{zu}$ ergänzen und dass im Einklang mit bestehenden Forschungsergebnissen zweitens ein klarer Fach- und Situationsbezug prädiktiv für erfolgversprechende Fortbildungskonzeptionen ist.

Schlüsselwörter Lehrerfortbildung · Forschung · Wirksamkeit · Fachbezug

T. Jaschke $(\bowtie)$

Pädagogische Hochschule Ludwigsburg, Ludwigsburg, Deutschland

E-Mail: jaschke@ph-ludwigsburg.de 


\title{
Effectiveness assumptions with respect to the teaching action at gradu- ates of a general educational teacher training
}

\begin{abstract}
In the recent years, the effectiveness of training teacher has become a big subject in empirical educational research. In this paper, an exploratory interview study $(N=3)$ will be presented, in which the graduates of a general educational teacher training report on the effectiveness of the program. The study examines the question about the impact of the teachers who participated in the General Education Teacher Training. The teaching action here is conceptualized as part of the field of teacher activity and operationalized in this study as a way of planning and carrying out the regular mathematics teaching of respondents. The statements of the interviewees pointed out primarily changes in the methodological area, while substantial substantive impact of the teacher training, in terms of planning and carrying, are hardly observed. Although the participants of the teacher training apparently note a "refreshed" awareness of their educational attitude, the independent transfer of training content in the subject teaching is recognizably problematic. Overall, the results suggest that it may be useful to complete general pedagogical trainings for teachers with a subject-specific supplement which, in accordance with existing research, results a clear technical and situational context that is predictable for successful training concepts.
\end{abstract}

Keywords Inservice teacher training $\cdot$ Research $\cdot$ Effectiveness $\cdot$ Subject reference

\section{Einleitung und Vorstellung der Forschungsfrage}

Im vorliegenden Beitrag wird eine explorative Vorstudie vorgestellt, in der ehemalige Teilnehmerinnen und Teilnehmer einer allgemeinpädagogischen Lehrerfortbildung Selbstauskünfte zu ihren Wirksamkeitsvermutungen geben. Untersucht wird die Frage, welche subjektiv wahrgenommenen Auswirkungen die absolvierte Lehrerfortbildung auf das Unterrichtshandeln der beteiligten Lehrpersonen hatte. Unterrichtshandeln wird dabei als Teilbereich des Lehrerhandelns konzeptualisiert und operationalisiert sich im Rahmen dieser Studie in Planung und Durchführung des mathematischen Regelunterrichts der Interviewten.

Ausgangspunkt der hier dargestellten Untersuchung ist die allgemeinpädagogische, modular ausgerichtete und primär auf die pädagogische Haltung der Lehrenden abzielende Weiterbildungskonzeption eVOCATIOn zur Professionalisierung von Lehrpersonen im Bereich der Potenzial- und Begabungsförderung. Die Ergebnisse sollen im Nachgang der hier dargestellten Vorstudie dazu verwendet werden, ein mathematikspezifisches Fortbildungsmodul zur Ergänzung der eVOCATIOn-Lehrerfortbildung zu entwickeln.

In Abschn. 2 werden zunächst empirische und theoretische Erkenntnisse zur Wirksamkeit von Lehrerfortbildungen und zur pädagogischen Haltung von Lehrkräften dargestellt, welche zum einen den Rahmen dieser Arbeit abstecken und zum anderen zur Einordnung und Verortung der Ergebnisse genutzt werden. Außerdem wird die dieser Arbeit zugrunde liegende allgemeinpädagogische Lehrerfortbildung des 
eVOCATIOn-Weiterbildungsinstituts skizziert und der für die Fortbildungskonzeption grundlegende pädagogische bzw. personorientierte Begabungsbegriff erläutert.

Das methodische Vorgehen der Studie inklusive des deduktiv-induktiv entwickelten Kategoriensystems zur Auswertung der qualitativen Interviews ist Inhalt des 3. Abschnittes. Im 4. Abschnitt werden dann zentrale Ergebnisse dargestellt und vor dem Hintergrund der aktuellen Forschungslage eingeordnet. Abschn. 5 fasst die Ergebnisse der Studie nochmals zusammen und diskutiert diese auf der Folie des theoretisch-empirischen Rahmens von Abschn. 2.

\section{Theoretisch-empirischer Rahmen}

\subsection{Empirische Erkenntnisse zur Wirksamkeit von Lehrerfortbildungen}

Im Anschluss an die „empirische Wende“ in der Erziehungswissenschaft ist in Deutschland die Lehrerbildung verstärkt in die Diskussion geraten. Nachdem auf Grundlage der Expertise von Terhart (2002) zunächst Standards für die erste und zweite Phase der Lehrerbildung formuliert wurden, wird in jüngerer Zeit auch zunehmend die dritte Phase in den Blick genommen (vgl. Müller et al. 2010). Aktuell ist nach Leuders (2012, S. 114) das Wissen um die Bedingungen und Wirkungen von Lehrerbildung geprägt von ,starken Überzeugungen und schwachen Evidenzen“ (vgl. dazu auch Yoon et al. 2007). Die zentrale Wirkungskette der Lehrerausund Weiterbildung Lehrerbildung $\rightarrow$ Lehrerkompetenzen $\rightarrow$ Lehrerhandeln $\rightarrow$ Schülerkompetenzen ist in vielen Bereichen noch wenig aufgeklärt und erfordert umfassende Forschungsanstrengungen (vgl. dazu auch die Ebenen der Wirksamkeit von Lehrerfortbildungen bei Lipowsky 2010). Klar ist, dass die einzelnen „Kettenelemente" nicht automatisch aufeinander folgen, so dass beispielsweise veränderte Lehrerkompetenzen nicht zwangsläufig verändertes Lehrerhandeln zur Folge haben. $\mathrm{Zu}$ stabil sind dazu die subjektiven Theorien geringer Reichweite, die mit ihren Situations- und Handlungsprototypen das praktische Lehrerhandeln bestimmen (vgl. Wahl 2013).

Obwohl die Determinanten gelingender Lehrerfort- und Weiterbildungen auch international noch unzureichend erforscht sind (vgl. Yoon et al. 2007), lassen sich auf Grundlage empirischer Forschungsergebnisse dennoch verschiedene Kriterien benennen, die den Erfolg von Lehrerfortbildungen offenbar maßgeblich beeinflussen (vgl. Reusser und Tremp 2008; Lipowsky 2010). Bezüglich der Wirksamkeit von Lehrerfortbildungen ist bekannt, dass Lehrerfortbildungen die in sie gesetzten Erwartungen und Ziele in der Regel kaum erfüllen können und nur selten zu nachhaltigen Transfereffekten führen (vgl. Reusser und Tremp 2008). Es lassen sich aber auch empirisch ermittelte Merkmale erfolgversprechender Fortbildungsformate identifizieren (vgl. Lipowsky 2010), von denen die Orientierung an ganz konkreten und fachbezogenen Lernhandlungen von Schülerinnen und Schülern von vielen Autoren betont wird (vgl. etwa Leuders 2012; Kunter et al. 2011; Lipowsky 2011; Reusser und Tremp 2008; Hattie 2014). Danach sollen die bei Lehrkräften vorhandenen Argumentationen, Vorstellungen, Haltungen und Einstellungen (beliefs), aber auch deren professionelles Wissen in Bezug auf Fachunterricht und Schülerlernen 
im Rahmen einer Fortbildung kritisch und reflektierend in den Blick genommen werden. Ziel einer Lehrerfortbildung muss es vor diesem Hintergrund sein, Lehrpersonen die relevante Beziehung zwischen dem unterrichtlichen Handeln (Lehrerhandeln) und dem Lernen der Schülerinnen und Schüler erkennbar zu machen (vgl. Lipowsky 2011). Dazu sollen sich Lehrerinnen und Lehrer primär mit dem Lernen und Verstehen der Lernenden beschäftigen. Methodisch wird z. B. die Analyse von Schülervorstellungen oder -fehlern in Verbindung mit Schülerprodukten oder eigenen und fremden Unterrichtsvideos (vgl. dazu Krammer et al. 2010) vorgeschlagen - und diese möglichst fallbezogen und orientiert an konkreten Unterrichtsthemen. Flexibles Handlungswissen soll also in weitgehend authentischen und sozialen Praxiskontexten erworben werden (vgl. dazu auch Wahl 2013).

\subsection{Pädagogische Haltung}

Schwer und Solzbacher (2014) arbeiten in ihrem Buch „Professionelle pädagogische Haltung" heraus, dass subjektive Überzeugungen, Werte und Normen von Lehrkräften erst dann in entsprechendes Verhalten umgesetzt werden, wenn sie mit den handlungsleitenden Kompetenzen verknüpft sind. Es reicht nicht aus, ,,einer pädagogischen Fachkraft positive Überzeugungen und Einstellungen kognitiv zu ,predigen“, zu ,vermitteln' oder diese zu fordern. Positive Überzeugungen helfen wenig, wenn sie nicht mit den entsprechenden objektiven Fähigkeiten verbunden sind" (Schwer und Solzbacher 2014, S. 83). Es ist sogar davon auszugehen, dass Erfahrungen in gelebter Unterrichtspraxis für den Aufbau und die Veränderung pädagogischer Haltungen entscheidend sind, da ,sich das Selbst in der und durch die Bewältigung von Herausforderungen in Alltag und Beruf herausbildet" (ebd., S. 55). Für das Unterrichtsfach Mathematik stellt Blömeke (2010, S. 219) fest, dass Lehrkräften in Deutschland zwar grundsätzlich eine hohe berufsethische Haltung bescheinigt werden kann, es in der Praxis aber offenbar nicht zufriedenstellend gelingt, diese in konkreten Unterrichtssituationen in adäquate Handlungen umzusetzen. Damit sich pädagogische Haltungen auf der Ebene des Lehrer- und Unterrichtshandelns manifestieren können, bedarf es also zwingend objektiv-fachdidaktischer Kompetenzen im Bereich des Professionswissens (vgl. Kunter et al. 2011; Ball et al. 2008; Shulman 1987). Auch in der SCHOLASTIK-Studie kommen Helmke und Weinert (1997) zu dem Schluss, dass es in erster Linie die Kompetenzen der Lehrkraft sind, die den größten Einfluss auf das Schülerlernen haben und somit den Persönlichkeitsmerkmalen vorgeordnet sind. Sind diese Kompetenzen nicht oder nur unzureichend vorhanden, ist davon auszugehen, dass sich bzgl. der fachdidaktischen Unterrichtsgestaltung (Unterrichtshandeln) nur geringe Auswirkungen der pädagogischen Haltung identifizieren lassen. Eine pädagogische Haltung ist letztlich also in erster Linie das Ergebnis bewältigter oder nicht-bewältigter Herausforderungen in der pädagogischen Praxis. Und ob herausfordernde Situationen im Unterrichtsalltag bewältigt werden oder nicht, hängt offenbar in entscheidender Weise von den jeweils vorhandenen objektiven Kompetenzen einer Lehrkraft ab. 


\subsection{Das eVOCATIOn-Weiterbildungsinstitut und der pädagogische Begabungsbegriff}

Das eVOCATIOn-Weiterbildungsinstitut ist aus einem Comenius-Projekt im Rahmen des von der Europäischen Union geförderten Sokrates-Programms „Lebenslanges Lernen“ hervorgegangen und bietet allgemeinpädagogische, nicht fachdidaktisch ausgerichtete Fort- und Weiterbildungen im Bereich Begabungs-, Leistungs- und Potenzialentwicklung an. eVOCATIOn zielt dabei auf die „Entwicklung personaler und methodischer Kompetenzen von Lehrkräften, die heterogene Begabungsprofile respektieren sowie in ihrem Unterricht den allgemeinen Prinzipien einer begabungsfreundlichen Lernumwelt folgen wollen“ (Gräbner und Cronenberg 2016). In insgesamt fünf Modulen erhalten Lehrkräfte durch Referenten aus Wissenschaft und Praxis eine Einführung in theoretische Grundlagen und handlungspraktische Gestaltungsformen einer personorientierten Pädagogik (vgl. Weigand 2014) sowie entsprechende allgemeindidaktische Konsequenzen für die Unterrichts- und Schulentwicklung. Die insgesamt auf anderthalb Jahre angelegte Weiterbildung sieht vor, dass die teilnehmenden Lehrkräfte von ihnen selbst ausgewählte Inhalte der Weiterbildung in ihrer eigenen schulischen Praxis erproben. Diese Erprobung findet während der sogenannten Praxisphasen in weitgehender Eigenverantwortung zwischen den einzelnen Modulen statt, wobei es die Möglichkeit der Begleitung durch einen eVOCATIOn-Referenten gibt. Die teilnehmenden Lehrpersonen reflektieren ihre Erfahrungen in den jeweils nachfolgenden Präsenzphasen.

Grundlage von eVOCATIOn ist ein pädagogischer Begabungsbegriff (Weigand 2014; Schenz 2011; Schwer und Solzbacher 2014), der im Unterschied zu multifaktoriellen Begabungsmodellen (Ziegler 2008, 2010) Begabungen als domänenspezifische Potenziale konzeptualisiert, die sich im schulischen Bereich in einem Prozess der Wechselwirkung mit vorhandenen Lerngelegenheiten entfalten. Der einzelne Schüler und die einzelne Schülerin werden als Subjekt seiner/ihrer Begabungen und Interessen betrachtet; und dies unabhängig von der aktuell gezeigten oder prognostizierten Leistung. Aufgabe von Lehrpersonen auf der Ebene des schulischen Regelunterrichts ist im Sinne des pädagogischen Begabungsbegriffs damit in erster Linie die Entwicklung und Gestaltung konkreter und lerninhaltsspezifischer Lerngelegenheiten. Nach Ladenthin (2006) erfordert ein begabungsorientierter Mathematikunterricht keine spezielle Didaktik oder Methodik, so dass die Lerngegenstände und die mit ihnen verbundenen Aneignungshandlungen prinzipiell für alle Lernenden gleich sind. Die Kriterien eines mathematikdidaktischen Qualitätsbegriffs können daher praktisch ohne Einschränkungen auf begabungsorientierten Mathematikunterricht übertragen werden (vgl. dazu auch Schenz et al. 2011).

\section{Methode}

Ausgangspunkt der im Folgenden dargestellten empirischen Untersuchung ist die Lehrerfortbildung des Weiterbildungsinstituts eVOCATIOn (vgl. http://www.ewib. de), die unter Abschn. 2.3 in groben Zügen beschrieben wurde. 
Die Entscheidung für eine konkrete Forschungsmethode muss stets von ihrer Frage- und Zielperspektive her begründet werden (Gegenstandsangemessenheit). Die oben formulierte Fragestellung legt dabei eine Befragung ehemaliger Teilnehmerinnen und Teilnehmer der eVOCATIOn-Weiterbildung nahe, da eine direkte Erfassung des Unterrichtshandelns beispielsweise durch Unterrichtsbeobachtungen aus forschungspraktischen und -ökonomischen Gründen nicht möglich war. In sogenannten Leitfadeninterviews (vgl. Stigler 2005) wurden daher im Frühjahr 2015 drei gymnasiale Mathematiklehrkräfte (als Tom, Hans und Anna anonymisiert) aus Bayern befragt, die in der jüngeren Vergangenheit an einem eVOCATIOn-Basiskurs teilgenommen haben. Interviewer war der Autor selbst, der die Befragten im Vorfeld weder kannte noch irgendeine Funktion in der Fortbildung innehatte. Es handelte sich bei den Interviewsituationen daher um reine Forschungssettings.

Da die Interviews neben einer Einschätzung der erlebten Auswirkungen der Weiterbildung auf der Ebene des Lehrer- und Unterrichtshandelns auch zu einer generellen Evaluation der eVOCATIOn-Weiterbildung genutzt wurden, wurde den Probanden nach einer Erklärung zu den Anforderungen und Besonderheiten eines wissenschaftlichen Interviews zunächst die Möglichkeit eröffnet, sich offen und frei strukturierend zu den Eindrücken, Erlebnissen und Auswirkungen der eVOCATIOnWeiterbildung zu äußern (vgl. Schütze 1983). Während der Äußerungen der Interviewpartner wurde vom Interviewer versucht, relevante und möglicherweise noch nicht klar genug erscheinende Aspekte schriftlich zu fixieren, so dass diese im Anschluss an die erste „Erzählphase“ der Probanden in einem Nachfrageteil nochmals vertiefend thematisiert werden konnten. Neben diesen vorab nicht planbaren Punkten wurden a priori auch einige speziellere Vertiefungsfragen in Form eines kleinen Interviewleitfadens (vgl. Stigler et al. 2005, S. 129; Mayring 2002) generiert, die insbesondere die konkreten unterrichtlichen Aspekte im Mathematikunterricht fokussierten. Im Einzelnen waren dies:

- Hat ihnen die eVOCATIOn-Fortbildung für ihren alläglichen Mathematikunterricht geholfen?

- Hat sich ihre Unterrichtsplanung durch die eVOCATIOn-Weiterbildung verändert?

- Hat sich die Durchführung ihres Mathematikunterrichts durch eVOCATIOn verändert?

- Welche Materialien und Umsetzungshilfen innerhalb der eVOCATIOn-Fortbildung wären aus ihrer Sicht für eine Umsetzung im Fach Mathematik möglicherweise hilfreich?

- Welche Wünsche hätten sie für eine fachspezifische eVOCATIOn-Fortbildung?

- Wie könnte man ihres Erachtens die eVOCATIOn-Weiterbildung im Hinblick auf Mathematikunterricht verbessern?

Die Interviews der Probanden wurden im Anschluss nach den Transkriptionsregeln von Dresing und Pehl (2011) mit dem Transkriptionsprogramm f4 transkribiert und durch eine inhaltlich strukturierende Inhaltsanalyse ausgewertet (vgl. Kuckartz 2014; Mayring 2010). Eine deduktiv-induktive Kategorienbildung (vgl. Kuckartz 2014, S. 69) ergab das nachfolgende Kategoriensystem, wovon für die hier untersuchte Fragestellung in erster Linie die (Sub-)Kategorien Auswirkungen/Unterricht, 
Fachbezogene Erwartungen und Wünsche sowie Fachbezogene Defizite interessant und relevant sind. Im nachfolgenden Ergebnisteil wird nun die Auswertung dieser Kategorien dargestellt.

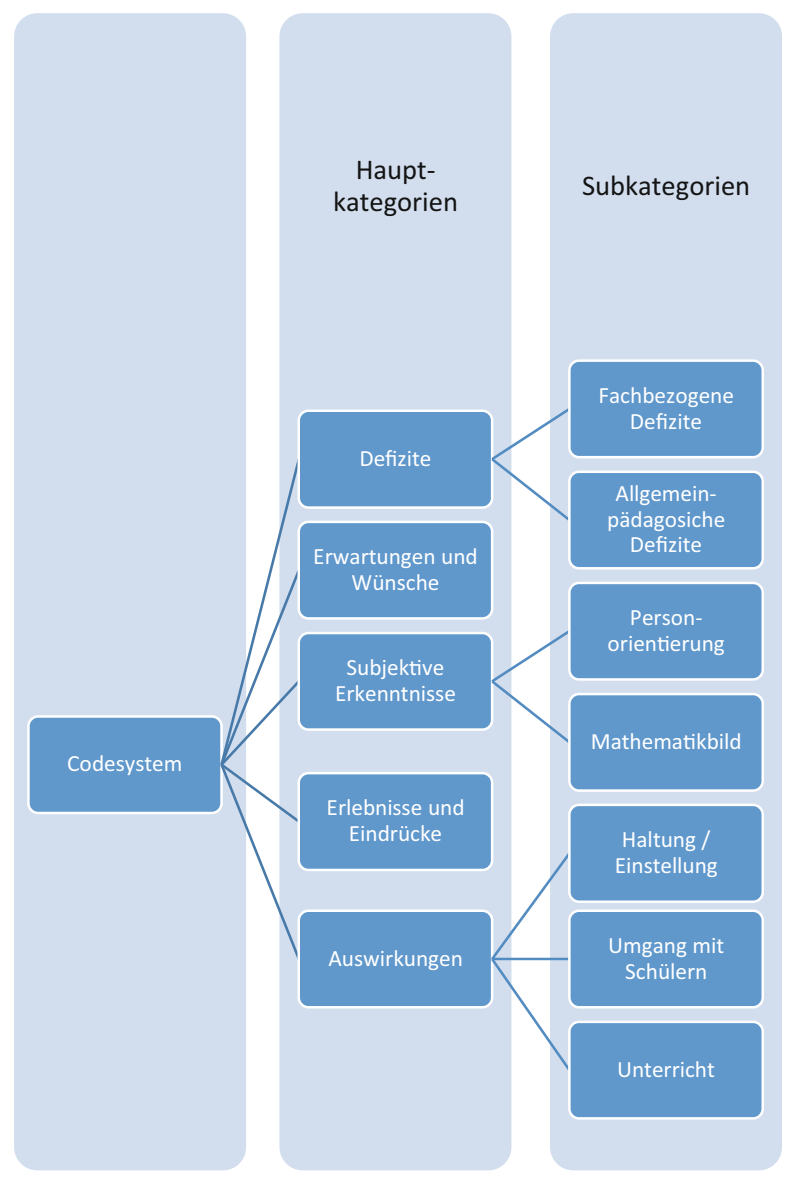

\section{Ergebnisse}

Die Ergebnisdarstellung der kategorienbasierten Auswertung erfolgt entlang der relevanten (Sub-)Kategorien Auswirkungen im Unterricht, Fachbezogene Erwartungen und Wünsche sowie Fachbezogene Defizite.

1. Kategorie: Auswirkungen im Unterricht

Bezüglich des Unterrichts lassen sich die Auswirkungen von eVOCATIOn primär im methodischen Bereich verorten. Alle befragten Lehrkräfte erklären, seit der Weiterbildung vermehrt selbstständige und organisatorisch-differenzierende Arbeitsformen einzusetzen sowie den Lernenden mehr Raum bei der methodischen Gestaltung des eigenen Lernprozesses zu geben. Während Hans versucht, das dialogische Lernen 
von Gallin und Ruf (2014) sowie die Idee der Lerntagebücher stärker in seinen Unterricht zu integrieren, arbeiten Tom und Anna vermehrt mit vorstrukturierten Selbstlernphasen, in denen sich Schülerinnen und Schüler eigenständig und primär nach Lerntempo differenziert das mathematische Wissen aneignen.

Anna: Vom Unterrichtsplan her gestalte ich den Unterricht denke ich deutlich freier. Also in Phasen auf jeden Fall. Ich denke im Mathematikunterricht sind viele Schüler darauf angewiesen, dass man auch mal den Stoff relativ eng darlegt. Das heißt, ich mache phasenweise engeren Unterricht und phasenweise wieder offeneren, wo sich die Schüler selber aussuchen können, wie viel sie arbeiten, wie sie mit den Lösungen umgehen, ob sie überhaupt dem Schulstoff folgen oder ob sie nicht lieber an Wettbewerbsaufgaben in einem Extraraum arbeiten wollen.

Tom: Habe dann also verschiedene Phasen, Selbstlernzeiten, Ende der fünften Klasse und jetzt auch in der sechsten Klasse mit Bruchrechnen dann mal wieder ausprobiert.

Im Gegensatz zu deutlich erkennbaren und prinzipiell fachunspezifischen Ergänzungen in den methodisch-organisatorischen Ansätzen der interviewten Lehrpersonen scheint die eVOCATIOn-Weiterbildung auf die Aufbereitung der Unterrichtsgegenstände und die inhaltlich-didaktische Gestaltung des Unterrichts nur beschränkten Einfluss gehabt zu haben. Lediglich Hans gibt an, das dialogische Lernen (vgl. Gallin und Ruf 2014), welches allerdings kein expliziter Inhalt des eVOCATIOnBasiskurses ist, auch zur problemorientierten Einführung neuer Unterrichtsinhalte zu nutzen. Ein kausaler Zusammenhang zwischen diesem Ansatz und der besuchten Weiterbildung ist bei Hans aber nur bedingt herstellbar, da er im Interview neben dem eVOCATIOn-Basiskurs noch diverse weitere Aspekte thematisiert, die ihn in der Vergangenheit offenbar beeinflusst haben. Allerdings hat die Weiterbildung bei Hans nach seinen Angaben dazu beigetragen, die unterschiedlichen Aspekte zu reflektieren und in einen Sinnzusammenhang zu bringen.

Für Anna und Tom hat die eVOCATIOn-Weiterbildung im Bereich der Einführung neuer Unterrichtsinhalte keine substanzielle Veränderung in Richtung eines lernerzentrierten, begabungsorientierten Unterrichts evoziert. Anna gibt sogar an, dass sie im Widerspruch zu mathematikdidaktischen Qualitätskriterien in den Begabtenklassen Unterrichtsinhalte nun deutlich schneller einführt und früher die grundlegenden Aufgaben rechnen lässt. Dabei erklärt sie den Lernenden im Sinne einer instruktionalen Aufgabendidaktik kurz die Grundidee und hat nach eVOCATIOn ein Skript, das schnellere Schülerinnen und Schülern zum Selbststudium nutzen können.

Anna: Jetzt Klasse 9M, einfach das Herleiten von der Mitternachtsformel. Also das hätte ich vor eVOCATIOn relativ lange an der Tafel mit der Klasse gemeinsam gemacht. Nach eVOCATIOn ist es so, dass ich das alles als Skript habe, den Schülern kurz die Grundidee erkläre, denjenigen sage, die eh schnell sind, schaut es euch an, es ist ganz witzig, wie man es machen kann. Und (...) während die das dann schnell angucken, erkläre ich den anderen das ein bisschen detaillierter und dann wird gerechnet. Sehr schön. 
Die unveränderten Unterrichtsplanungen werden bei Anna an folgenden Interviewpassagen bzw. Zitaten deutlich:

I: Und wenn es darum geht, Unterrichtsinhalte einzuführen beispielsweise. Haben sie dann in irgendeiner Form eine Veränderung gemerkt? Dass sie z. B. denken, ja gut, früher habe ich es so und so gemacht und jetzt muss vielleicht eine Einführung anders laufen vor dem Hintergrund dessen, was bei eVOCATIOn gesagt worden ist?

Anna: Nein, das kann ich nicht beobachten. Bei mir nicht beobachtbar. Kann aber auch einfach heißen, dass ich halt da ...

I: Muss auch nicht sein.

Anna: Nein, nein. Ist auch nicht. Deswegen musste ich so lange überlegen, weil ich denke, dass ich da zwar Sachen modifiziere, aber das ist nicht aus eVOCATIOn-Gründen, sondern einfach weil ich dann mit meinem eigenen einführenden Beispiel nicht mehr so zufrieden bin. Also das ist eher inhaltlich, mathematisch dann.

Bei Tom hört sich das so an:

Tom: Aber so meinen Grobplan und den Detailplan auch für den restlichen Bereich (außer der Planung der Selbstlernphase) habe ich eigentlich nicht geändert, so dass ich eigentlich sagen muss, da hat es eigentlich auch keinen Einfluss gehabt, würde ich sagen.

Insgesamt reduzieren sich die Auswirkungen der eVOCATIOn-Weiterbildung auf der Ebene des Unterrichtshandelns eher auf den methodischen Bereich. In manchen Unterrichtsphasen wird das gemeinsame Lernen durch vorbereitete und vorstrukturierte Unterrichtsmaterialien aufgelöst und individualisiert. Eine veränderte fachdidaktisch-inhaltliche Unterrichtsgestaltung ist kaum erkennbar.

2. Subkategorie: Fachbezogene Defizite

Recht übereinstimmend weisen die Interviewpartner darauf hin, dass die Weiterbildung in vielen Bereichen zwar zu Denkanstößen geführt hat, insgesamt aber offenbar $\mathrm{zu}$ abstrakt und allgemein angelegt war und konkrete Umsetzungshilfen gefehlt haben.

Anna drückt dies so aus:

Anna: Dass wir zwar sehr viele Anregungen bekommen haben, in welcher Form kann Schule entwickelt werden, in welcher Form kann personorientierter Unterricht ablaufen, aber alles auf einem sehr abstrakten, interessanten, aber auf einem sehr abstrakten Niveau. Das wäre, da komme ich eigentlich jetzt schon dazu zu sagen, das ist eine der Sachen, die wahrscheinlich gefehlt haben, bzw. eine der Sachen, wo man jetzt von der Anregung her sagen muss, man kommt dann an seine eigene Schule zurück und überlegt sich eher dann wieder konkreter werdend, was bedeutet das für die Organisation wirklich in 
meinem Fachbereich, für die Organisation oder die Gespräche mit den Kollegen, für die Teamarbeit und wirklich auch für den Fachunterricht.

Neben der abstrakten Fortbildungsanlage beklagten die befragten Lehrkräfte das Fehlen fachbezogener Einheiten und Konkretisierungen. So kann sich Anna etwa daran erinnern, ,dass auch damals schon vor allem angesprochen wurde, dass fachbezogene Einheiten gefehlt haben". Und Tom stellt fest, dass es viele Anregungen gibt, ,die man schon irgendwie auf den Unterricht beziehen kann. Ohne dass sich dabei während der Fortbildung was Konkretes eigentlich draus entwickelt, was man konkret im Unterricht umsetzen kann, was man anders machen kann, was man anders gestalten kann “. Für Tom hat sich der größere Rahmen, den eVOCATIOn vermitteln will ,noch nicht konkret mit Leben gefüllt “. Er ist nach wie vor der Weiterbildung auf der Suche nach sinnstiftenden Inhalten, deren Relevanz er sich schon vor der Veranstaltung bewusst war. Darüber hinaus hat sich sein lernprozessorientiertes Feedback an Lernende durch die Weiterbildung nicht verändert.

\section{Subkategorie: Fachbezogene Erwartungen und Wünsche}

Die von den Probanden in den Interviews formulierten Erwartungen und Wünsche leiten sich größtenteils aus den diagnostizierten Fehlstellen der Weiterbildung ab. Anna wünscht sich gute Ideen für konkrete Aufgaben und Möglichkeiten der Unterrichtsentwicklung. So schlägt sie beispielsweise für die Weiterbildung vor, „Ideen $z u$ entwickeln (...), welche Themengebiete in der fünften, in der sechsten Klasse mit den Kindern gewinnbringend umgesetzt werden können. Also was die bauen können, wo sie rechnen können, wo sie vielleicht selbst forschen können “. Wie Anna wünscht sich auch Tom konkrete Positivbeispiele für die unterrichtliche Realisierung personorientierten Mathematikunterrichts. Die Weiterbildung darf sich seiner Meinung nach nicht im Postulat bestimmter Unterrichtsprinzipien und Wertvorstellungen erschöpfen, sondern muss auf die reale und fallbezogene Situation des Fachunterrichts transformiert werden.

Tom: Nicht nur die Begründung, warum es wichtig ist; die war sehr gut und das war auch notwendig quasi, diesen Weg mal zu gehen, in Gedanken zumindest. Aber wie ich das dann mache, damit waren wir wieder alleine quasi. Die Gedanken hat sich noch keiner gemacht. Soweit wurde der Faden noch nicht gesponnen. Aber das würde ich mir schon wünschen. (...) Also das ist natürlich schwierig, aber die Prinzipien, die sich aus diesem eVOCATIOn erwachsen, die müssten ja jetzt auf einen Unterricht hinauslaufen. Wie soll dieser typische Unterricht aussehen? Und idealerweise würde man dafür - vielleicht nicht den fertigen Unterricht, aber zumindest müsste man sich überlegen, welche Punkte wären uns wichtig didaktisch und wie konkret könnte man das umsetzen.

Um den Unterricht im Sinne von eVOCATIOn zu entwickeln, schlägt Tom für potenzielle Mathematikfortbildungen vor, Sequenzen einer Unterrichtsstunde kooperativ zu entwickeln.

Tom: Und da kann man auch schön sich Gedanken machen zu sinnstiftenden Inhalten und was ich quasi so gewinnreich daran finden würde ist, man lernt 
in diesem Prozess mit den anderen, wie man das denn machen müsste, wenn man zu Hause vor dem Problem sitzt und die Motivation hat, sich jetzt damit zu beschäftigen, wie kann ich für die nächste Unterrichtseinheit das im Sinne von eVOCATIOn vielleicht umsetzen. Also gar nicht dieser fertige Inhalt, sondern dass man bisschen lernt, Inhalte selbst aufzubereiten.

\section{Zusammenfassung der Ergebnisse und Diskussion}

Ausgangspunkt der dargestellten Interviewstudie war die Fragestellung, welche subjektiv wahrgenommenen Auswirkungen die absolvierte Lehrerfortbildung auf das Unterrichtshandeln der beteiligten Lehrpersonen hatte.

Mit Blick auf das konkrete fachdidaktisch-unterrichtliche Lehrerhandeln (Unterrichtshandeln) weisen die Ausführungen der Interviewpartner primär auf punktuelle Veränderungen im methodisch-organisatorischen Bereich hin. Substanzielle didaktisch-inhaltliche Auswirkungen auf die Unterrichtsplanung und -durchführung sind hingegen kaum zu beobachten. Die Befragten geben übereinstimmend an, dass die eVOCATIOn-Weiterbildung zwar zu einer reaktivierten Sensibilisierung in Bezug auf Personorientierung und den pädagogischen Begabungsbegriff geführt hat, diese aber in der Praxis nur schwer konkretisierbar ist. Gefehlt haben nach Angaben der Interviewten v. a. fachbezogene Einheiten zum Transfer und zur Anwendung der theoretischen Fortbildungsinhalte auf bzw. im ,normalen Unterricht“. Man kann daraus folgern, dass es offenbar nicht ausreicht, mentale Inhalte im Rahmen von Fortbildungsveranstaltungen lediglich zu ,vermitteln“ oder zu fordern, da die eigenständige Umsetzung pädagogischer Haltungen, Wertvorstellungen und Überzeugungen in der Praxis mit großen Schwierigkeiten verbunden zu sein scheint (vgl. dazu auch Schwer und Solzbacher 2014). Notwendig erscheinen daher Fortbildungselemente, die theoretische Konstrukte wie den pädagogischen Begabungsbegriff so aufbereiten und für die Teilnehmerinnen und Teilnehmer so reduzieren und konkretisieren, dass deren Realisierung im Unterrichtsalltag tatsächlich gelingen kann. Denn nur auf Basis einer gelingenden Praxis und eigenem Kompetenzerleben kann sich in der Folge eine Veränderung der pädagogischen Haltung einstellen - nicht umgekehrt! Auf Basis der Ergebnisse dieser Vorstudie ist es ein Trugschluss zu glauben, unterrichtliche Realität könne alleine durch die Präsentation geeigneter Wertvorstellungen oder didaktischer Prinzipien verändert werden. Ansatzpunkt und Zielperspektive fachunterrichtlicher Weiterentwicklung sollte vielmehr das fachdidaktische Professionswissen (vgl. Kunter et al. 2011) der Lehrkräfte sein. Für die Lehrerfortbildung des eVOCATIOn-Weiterbildungsinstituts kann auf Grundlage dieser Studie die Empfehlung ausgesprochen werden, die derzeitige Fortbildungsstruktur durch fachspezifische Module zu ergänzen.

Zusammenfassend kann aufgrund der Ergebnisse gesagt werden, dass ein klarer Fach- und Situationsbezug, wie er von vielen Autoren bereits gefordert wird (vgl. Abschn. 2.1 sowie auch Barzel und Selters 2015), in der Tat ein entscheidender Prädiktor für die Wirksamkeit von Lehrerfortbildungen auf der Ebene des Lehrerhandelns zu sein scheint. Bleibt dieser jedoch aus, ist mit Wahl (2013) davon auszugehen, dass lediglich träges Wissen generiert wird, welches aufgrund der 
Dominanz jahrelang erfolgreich erprobter Handlungsmuster (subjektive Theorien geringer Reichweite) nicht handlungsleitend werden kann.

Open Access Dieser Artikel wird unter der Creative Commons Namensnennung 4.0 International Lizenz (http://creativecommons.org/licenses/by/4.0/deed.de) veröffentlicht, welche die uneingeschränkte Nutzung, Verbreitung und Wiedergabe für beliebige Zwecke erlaubt, sofern Sie den/die ursprünglichen Autor(en) und die Quelle ordnungsgemäß nennen, einen Link zur Creative Commons Lizenz beifügen und angeben, ob Änderungen vorgenommen wurden.

\section{Literatur}

Ball, D., Thames, M., \& Phelps, G. (2008). Content knowledge for teaching. What makes it special? Journal of Teacher Education, 59(5), 389-407.

Barzel, B. \& Selter, C. (2015). Die DZLM-Gestaltungsprinzipien für Fortbildungen. J Math Didakt. DOI: 10.1007/s13138-015-0076-y.

Blömeke, S. (2010). Kompetenzen deutscher Mathematiklehrer im internationalen Vergleich. Zentrale Ergebnisse der internationalen Vergleichsstudie TEDS-M. Humbold-Universität Berlin. https://www. teds-unterricht.uni-hamburg.de/bilder/teds-m-zusammenfassung.pdf. Zugegriffen: 25.1.2016.

Dresing, \& Pehl, T. (2011). Praxisbuch Transkription. Regelsysteme, Software und praktische Anleitungen für qualitative ForscherInnen. Marburg: Eigenverlag.

Gräbner, J., \& Cronenberg, U. (2016). Vorstellung des eVOCATIOn-Projekts. http://www.ewib.de/?page_ $\mathrm{id}=21$. Zugegriffen: 25.1.2016.

Helmke, A., \& Weinert, F.E. (1997). Entwicklung im Grundschulalter. Weinheim: Beltz.

Hattie, J. (2014). Lernen sichtbar machen. Baltmannsweiler: Schneider.

Krammer, K., Schnetzler, C. L., Pauli, C., Reusser, K., Ratzka, N., Lipowski, F., \& Klieme, E. (2010). Unterrichtsvideos in der Lehrerfortbildung. Überblick über Konzeption und Ergebnisse einer einjährigen netzgestützen Fortbildungsveranstaltung. In F. Müller, A. Eichenberger, M. Lüders, \& J. Mayr (Hrsg.), Lehrerinnen und Lehrer lernen. Konzepte und Befunde zur Lehrerfortbildung (S. 227-243). Münster: Waxmann.

Kuckartz, U. (2014). Qualitative Inhaltsanalyse. Methoden Computerunterstützung. Weinheim: Beltz Juventa: Praxis.

Kunter, M., Baumert, J., Blum, W., Klusmann, U., Krauss, S., \& Neubrand, M. (Hrsg.). (2011). Professionelle Kompetenz von Lehrkräften. Ergebnisse des Forschungsprogramms COACTIV. Münster, New York, München, Berlin: Waxmann.

Ladenthin, V. (2006). Brauchen Hochbegabte eine eigene Didaktik? In C. Fischer, \& H. Ludwig (Hrsg.), Begabtenförderung als Aufgabe und Herausforderung für die Pädagogik (S. 46-65). Münster: Aschendorff.

Leuders, T. (2012): Lehrerausbildung in Baden-Württemberg. Historische Entwicklungslinien und aktuelle Herausforderungen. In C. Cramer, K.-P. Horn, \& F. Schweitzer (Hrsg.), Lehrerausbildung in BadenWürttemberg. Historische Entwicklungslinien und aktuelle Herausforderungen (S. 115-146). Jena: IKS Garamond.

Lipowsky, F. (2010). Lernen im Beruf. Empirische Befunde zur Wirksamkeit von Lehrerfortbildungen. In F.H. Müller, A. Eichenberger, M. Lüders, \& J. Mayr (Hrsg.), Lehrerinnen und Lehrer lernen. Konzepte und Befunde zur Lehrerfortbildung (S. 51-70). Münster: Waxmann.

Lipowsky, F. (2011). Theoretische Perspektiven und empirische Befunde zur Wirksamkeit von Lehrerfortund -weiterbildung. In E. Terhart, H. Bennewitz, \& M. Rothland (Hrsg.), Handbuch der Forschung zum Lehrerberuf (S. 398-417). Münster, München, Berlin u. a.: Waxmann.

Mayring, P. (2002). Einführung in die qualitative Sozialforschung. Eine Anleitung zu qualitativem Denken (5., neu ausgestattete Aufl.). Weinheim: Beltz.

Mayring, P. (2010). Qualitative Inhaltsanalyse. Grundlagen und Techniken (11., aktual. überarb. Aufl.). Weinheim: Beltz.

Müller, F.H., Eichenberger, A., Lüders, M., \& Mayr, J. (Hrsg.). (2010). Lehrerinnen und Lehrer lernen. Konzepte und Befunde zur Lehrerfortbildung. Münster: Waxmann.

Reusser, K., \& Tremp, P. (2008). Diskussionsfeld „Berufliche Weiterbildung von Lehrpersonen“. Beiträge zur Lehrerbildung, 26(1), 5-10.

Ruf, U., \& Gallin, P. (2014). Austausch unter Ungleichen. Grundzüge einer interaktiven und fächerübergreifenden Didaktik (5. Aufl.). Seelze: Klett Kallmeyer Friedrich Verlag. 
Schenz, C., Rosebrock, S., \& Soff, M. (2011). Von der Begabtenförderung zur Begabungsgestaltung. Vom kreativen Umgang mit Begabungen in der Mathematik. Münster, Berlin: Lit.

Schütze, F. (1983). Biographieforschung und narratives Interview. Neue Praxis, 13(3), 283-293.

Schwer, \& Solzbacher, C. (2014). Professionelle pädagogische Haltung. Bad Heilbrunn: Klinkhardt.

Shulman, L.S. (1987). Knowledge and teaching: Foundations of the new reform. Harvard Educational Review, 57, 1-22.

Stigler, H. (2005). Praxisbuch empirische Sozialforschung in den Erziehungs- und Bildungswissenschaften. Wien, Bozen: Studien Verlag: Innsbruck.

Terhart, E., \& Reisch, L. (2002). Nach PISA. Bildungsqualität entwickeln. Hamburg: Europäische VerlagsAnstalt.

Wahl, D. (2013). Lernumgebungen erfolgreich gestalten. Vom trägen Wissen zum kompetenten Handeln; mit Methodensammlung (3. Aufl.). Bad Heilbrunn: Klinkhardt.

Weigand, G., Hackl, A., Müller-Oppliger, V., \& Schmid, G. (2014). Personorientierte Begabungsförderung. Eine Einführung. Weinheim: Beltz

Yoon, K. S., Duncan, T., Wen-Yu Lee, S., Scarloss, B., \& Shapley, K. L. (2007). Reviewing the evidence on how teacher professional development affects student achievement. http://ies.ed.gov/ncee/edlabs/ regions/southwest/pdf/REL_2007033.pdf. Zugegriffen: 25.1.2016.

Ziegler, A. (2008). Hochbegabung. Stuttgart: UTB.

Ziegler, A. (2010). Hochbegabte und Begabungsförderung. In R. Tippelt (Hrsg.), Handbuch Bildungsforschung (3., durchges. Aufl.) (S. 937-951). Wiesbaden: VS. 